\title{
Flash chromatography guided fractionation and antibacterial activity studies of Angelica archangelica root extracts.
}

\author{
Rayees A. Rather ${ }^{1,2}$, Shakeel-u-Rehman ${ }^{1}$, Syed Naseer ${ }^{1}$, Shabir H .Lone ${ }^{1}$, \\ Khursheed A. Bhat ${ }^{1}$, Anuja Chouhan ${ }^{2}$ \\ ${ }^{1,2}$ (Indian Institute of integrative Medicine, Sanatnagar Srinagar India-190005) \\ ${ }^{2}$ (Department of Chemistry Arni University, Kathgarh Indora (H.P) India-176401)
}

\begin{abstract}
Flash chromatography of Methanol, Methanol Water (1:1) and Water extracts of the roots of Angelica archangelica was carried out. The various fractions obtained were subjected to antibacterial activity against four clinically significant strains using Ciprofloxacin (antibiotic) as standard with fractions from methanol extract showing promising activity. One more interesting result was that all the ethyl acetate fractions from Methanol, Methanol Water (1:1) and Water extracts were more active. So these fractions can be further phytochemically investigated for the isolation of biologically active constituents. The positive biological activities manifested by the fractionated extracts from crude extracts render concrete substantiation for the use of such species for traditional medicinal purposes.
\end{abstract}

Keywords- Angelica archangelica, Antibacterial activity, Flash chromatography, Fractionation, MIC

\section{Introduction}

Production of antimicrobial secondary metabolites by maximum aromatic plants is as a response to pathogen attack and abiotic stress, in many cases these substances serve as plant defence mechanism against predation by microorganisms [1]. Angelica archangelica belonging to same category by its long history as folk medicine can be used for production of high efficacy drugs.

Angelica archangelica is one of the most respected medicinal herbs in Northern countries where it was cultivated during middle Ages [2]. Roots and seeds of this species are used as carminative, diaphoretic, diuretic, antiseptic and antidepressant. Roots are also used in the treatment of leucoderma and for dental preparation [34]. Tea made from roots of $A$. archangelica has been used as a folk remedy for stomach cancer [5]. Root of Angelica archangelica is also known as women ginseng in south west Asia and is used to treat amenorrhea, dysmenorrhea, menopausal disorders hypertension, anaemia and vegetovascular dystonia.

In India it grows wild in NW Himalayas of Kashmir and Garwal region (1500-3900 m asl). The whole herb is reported to be useful as stimulant, cordial, appetizer, dyspepsia, cardioactive, carminative, expectorant, diaphoretic and also in stomach troubles, billious complaints, infantile atrophy, menorrhiza, for treating rinderpest and constipation, etc. [6-10]

Phytochemistry of genus Angelica reveals presence of coumarins, mano and sesiquiterepenes such as angelicin, bergapten, imperatorin, isoimperatorin, xanthotoxin, oxypeucedanin [11]. Phytochemistry of other species of this genus also reveal some intresting compounds as Angelica dahurica root reveals pressance of 5,8 di-(2,3 dihydroxy-3 methylbutoxy)-psoralen along with ferulic acid[12]. Similar coumarins like heraclenin were isolated from Angelica lucida with interesting antimicrobial profile [13]. Bioactivity guided fractionation of chloroform extract from the roots of Angelica sinensis led to the isolation of (Z)-liguistilide, (E)-3-butylidene phthalide and (Z)-3-butyledene phthalide with prominent mosquito deterrent activity [16]. Imperatorin and Xantotoxin isolated from fruit of Angelica archangelica proved to be highly antiproliferative [14].

Based on the literature and phytochemistry of genus Angelica and very little published data concerning the content of biologically active substances in Angelica archangelica from North Western Himalayas of Kashmir region, with quite different climatic conditions that may influence the chemical composition of the plant, the current work was taken up. We followed a unique strategy to explore the bioactive components from Angelica archangelica. Fractionation of extracts of Angelica archangelica in different solvent systems through flash chromatography provides a route through which we can concentrate on the most active fractions for the isolation of biologically active molecules.

\section{Results and Discussion}

Various fractions of different Angelica archangelica extracts prepared through flash chromatography fractionation were subjected to antibacterial activity against four bacterial strains. The shade dried root of Angelica archangelica $(270 \mathrm{~g})$ was chopped, grinded and divided into three equal parts of $90 \mathrm{~g}$ each. One part was soaked in methanol and labelled as A001, second part was soaked in 1:1 methanol and water and labelled as 
A002 and the remaining part was soaked in water and labelled as A003. Each part was extracted four times at room temperature and concentrated under reduced pressure to give $11.50 \mathrm{~g}$ of A001, $16.75 \mathrm{~g}$ of A002 and 20.25 $\mathrm{g}$ of $\mathrm{A} 003$, in percentage terms of $13.85 \%$ for A001, $18.62 \%$ for $\mathrm{A} 002,22.5 \%$ for A003.

Fractionation of said crude extracts was carried through flash chromatography according to the standard flash chromatography protocols which are as under:

\subsection{1: Fractionation of A001:}

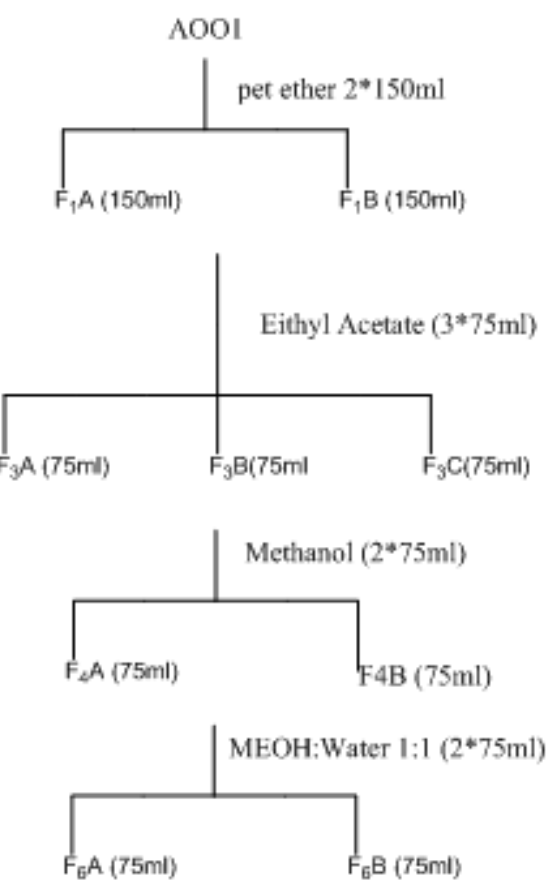

TOTAL NINE FRACTIONS

Result: A001-F3B $=0.27 \mathrm{~g}, \mathrm{~A} 001-\mathrm{F} 4 \mathrm{~A}=0.15 \mathrm{~g}, \mathrm{~A} 001-\mathrm{F} 4 \mathrm{~B}=1.02 \mathrm{~g}, \mathrm{~A} 001-\mathrm{F} 6 \mathrm{~A}=1.10 \mathrm{~g}, \mathrm{~A} 001-\mathrm{F} 6 \mathrm{~B}=0.25 \mathrm{~g}$

\subsection{2: Fractionation of A002:}
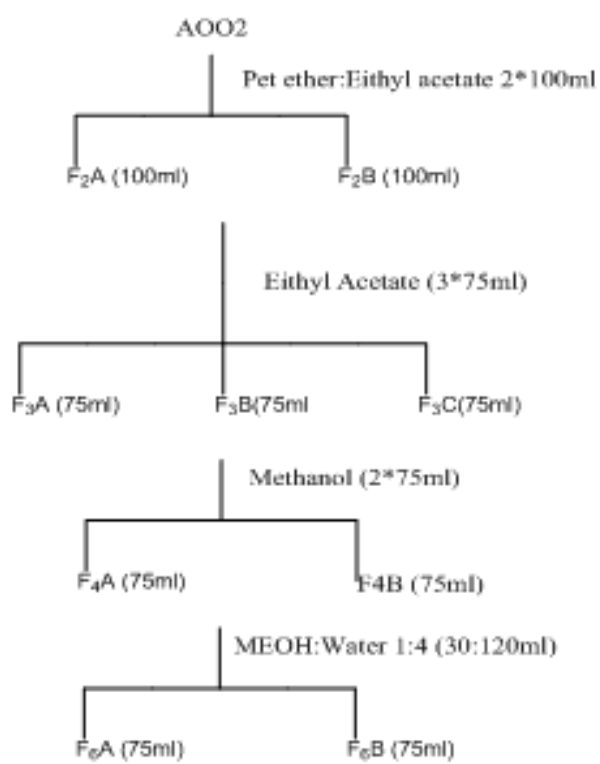

TOTAL NINE FRACTIONS

Result: $\mathrm{A} 002-\mathrm{F} 3 \mathrm{~A}=0.30 \mathrm{~g}, \mathrm{~A} 002-\mathrm{F} 3 \mathrm{~B}=0.50 \mathrm{~g}, \mathrm{~A} 002-\mathrm{F} 4 \mathrm{~B}=1.67 \mathrm{~g}, \mathrm{~A} 002-\mathrm{F} 6 \mathrm{~A}=0.25 \mathrm{~g}$ 


\subsection{3: Fractionation of A003:}
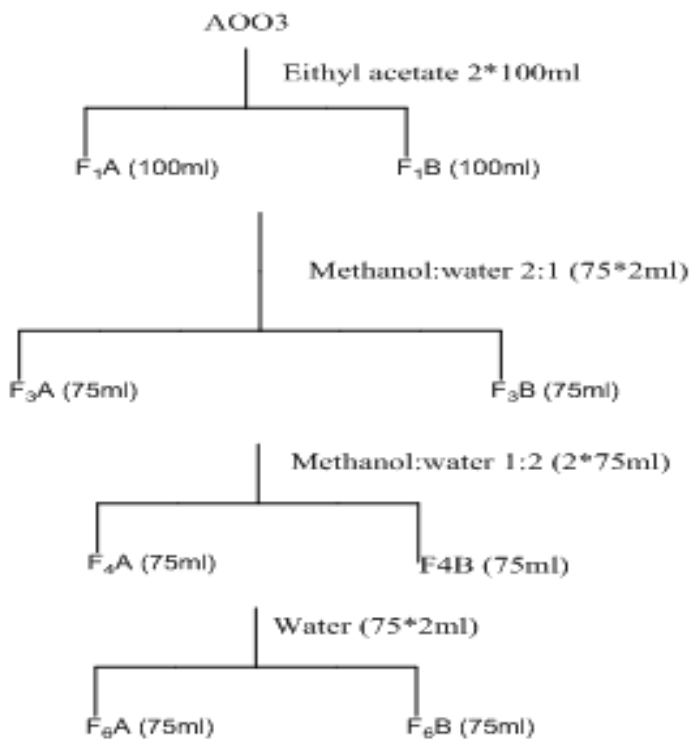

TOTAL EIGHT FRACTIONS

Result: A003-FIA= 0.30g, 003-F3A = 0.71g, A003-F4A=0.50g, A003-F4B=0.83g, A003-F6A = 0.43g

All these fractions were analyzed for their antimicrobial activity.

\section{2: Antimicrobial profile of fractionated extracts:}

The antimicrobial activity of the fractions expressed as minimum inhibitory concentration, are shown in Table 1. These extracts showed a broad range of antimicrobial activity against tested pathogens. The extracts were screened for antibacterial activity against Staphylococcus aureus, methicillin resistant Staphylococcus aureus Escherichia coli, Pseudomonas aeruginosa using micro-dilution method against the reference compound ciprofloxacin. F3b (A001), F4b (A001), F6a (A001), F3a (A002) and F3a (A003) were the most active fractions out of all the extracts tested. These five fractions exhibited MIC ranged from $125-500 \mu \mathrm{g} / \mathrm{ml}$ against the tested pathogens. From the above data it can be concluded that fractions from A001 extract show better antibacterial activity than the corresponding A002 and A003 extracts. One more interesting observation obtained from the antibacterial activity profile is that ethyl acetate fractions of $\mathrm{AOO} 1, \mathrm{AOO} 2$ and $\mathrm{AOO} 3$ were the most active.

TABLE 1: Antibacterial activity of the fractions from various extracts of A. Archangelica

\begin{tabular}{|c|c|c|c|c|c|}
\hline \multirow[t]{2}{*}{ S.No } & \multirow[t]{2}{*}{ Extract } & \multicolumn{4}{|c|}{$\mathrm{MIC}(\mu \mathrm{g} / \mathrm{ml})$} \\
\hline & & $\begin{array}{l}\text { S.aureus ATCC- } \\
25922\end{array}$ & $\begin{array}{l}\text { MRSA ATCC- } \\
15187\end{array}$ & $\begin{array}{l}\text { E.coli ATCC- } \\
25922\end{array}$ & $\begin{array}{l}\text { P.aeruginosa } \\
\text { ATCC-27853 }\end{array}$ \\
\hline 1 & F3b (A001) & 125 & 125 & 125 & 500 \\
\hline 2 & F4a (A001) & 1000 & 1000 & 1000 & 1000 \\
\hline 3 & F4b (A001) & 125 & 500 & 125 & 500 \\
\hline 4 & F6a (A001) & 125 & 250 & 125 & 250 \\
\hline 5 & F6b (A001) & 1000 & 1000 & 1000 & 1000 \\
\hline 6 & F3a (A002) & 125 & 125 & 500 & 500 \\
\hline 7 & F3b (A002) & 250 & 250 & 250 & 250 \\
\hline 8 & F4b (A002) & 250 & 250 & 500 & 500 \\
\hline 9 & F6a (A002) & 500 & 1000 & 1000 & 500 \\
\hline 10 & F1a (A002) & 1000 & 1000 & 1000 & 1000 \\
\hline 11 & F3a (A003) & 125 & 125 & 250 & 125 \\
\hline 12 & F4a (A003) & 500 & 500 & 125 & 500 \\
\hline 13 & F4b (A003) & 1000 & 1000 & 1000 & 500 \\
\hline 14 & F6a (A003) & 500 & 250 & 250 & 500 \\
\hline & Ciprofloxacin & 0.13 & 8.2 & $<0.03$ & 0.07 \\
\hline
\end{tabular}




\subsection{Plant material}

\section{Experimental}

The root parts of A. archangelica L. were collected in dominant stage in Feb 2012 from main campus of Indian institute of integrative medicines Sanatnagar Srinagar Kashmir (India). The plants were identified and authenticated by the taxonomist.

\subsection{Extraction and isolation procedure}

Fresh plant material (Roots) of Angelica Archangelica was dried in the oven set at $30^{\circ} \mathrm{C}$. The yield of extractives is increased by the pre-drying of the plant material as this leads to rupture of the cell structure and subsequently improved solvent access [15]. Once dried the plant material was powdered using a grinder. This powdered material was subjected to solvent extraction in three separate flasks, one with methanol labelled as (A001), another with methanol and water (1:1) labelled as (A002) and the third one with distilled water labelled as (A003) for 24 hours at room temperature. The extracted material and solvent were filtered (Whatman ${ }^{\circledR}$ filter paper no 41 , pore size $20-25 \mu \mathrm{m}$ ) to remove any plant material debris. Following this, the plant material was rinsed thrice with respective solvents and filtered after each rinse. The extracts were reduced under vacuum using a rotary evaporator (Büchirotavapor R-210, water bath B-491) at $45{ }^{\circ} \mathrm{C}$ for methanol and $60{ }^{\circ} \mathrm{C}$ for methanol water. The resultant extracts once concentrated to dryness were kept refrigerated prior to screening for biological activity.

\subsection{Flash chromatography fractionation.}

All the three crude extracts prepared i.e. A001, A002 and A003 were fractionated on Yemazen Corporation flash chromatogram with UV detector. Column was loaded with $8.0 \mathrm{gm}$ slurry $(3 \mathrm{~g}$ extract $+5 \mathrm{~g}$ silica gel) for each A001, A002 and A003 in 25 gm of silica gel (200-400 mesh size from Loba chemicals).The fractionated extracts were concentrated on buchi roto evaporators (R-210 water bath B-491).

\section{4: Medium used:}

Muller Hinton Agar and Muller Hinton Broth (Becton-Dickinson, Cockeysville, MD, USA; DIFCO laboratories).

\section{5: Bacterial strains, culture conditions and Antibiotics.}

The compounds were tested against following bacteria: Gram positive Staphylococcus aureus (ATCC25922), MRSA (ATCC-15187; Gram negative Escherichia coli (ATCC-25922) and Pseudomonas aeruginosa (ATCC-27853) obtained from American Type Cultures Collection (Manassas, VA, USA). The cultures were maintained on Tryptone soya agar and stored at $-70{ }^{\circ} \mathrm{C}$ containing $50 \%$ glycerol. Antibiotic was obtained from sigma -Aldrich, Ciprofloxacin was used as a standard antibacterial agent for this study. Stock solution was prepared at $1 \mathrm{mg} / \mathrm{ml}$.

\section{6: Minimum inhibitory concentration assay:}

MIC was determined as the guidelines of Clinical and Laboratory Standards Institute (Formerly the National Committee for Clinical Laboratory Standards) (Clinical and Laboratory Standards Institute, 2006). Bacterial suspensions were prepared by suspending $18 \mathrm{~h}$ grown bacterial culture in sterile normal saline. The turbidity of the bacterial suspension was adjusted to $0.5 \mathrm{McFarland}$ standards (equivalent to $1.5 \times 10^{8} \mathrm{CFU} / \mathrm{ml}$ ) at wavelength $625 \mathrm{~nm}$. The 2-fold serial of compounds $(1,2$ and 3, stock solution prepared in Dimethyl Sulphoxide) were prepared in Mueller Hinton Broth (MHB; DIFCO laboratories) in $100 \mu$ l volume in 96-well U bottom micro litre plates (Tarson, Mumbai, India). The above mentioned bacterial suspension was further diluted in the MHB and $100 \mu$ l volume of this diluted inoculum was added to each well of the plate resulting in the final inoculum of $5 \times 10^{5} \mathrm{CFU} / \mathrm{ml}$ in the well and the final concentrations of compounds ranged from 2000 to $3.90 \mu \mathrm{g} / \mathrm{ml}$ till $10^{\text {th }}$ column. Column 11 and column 12, containing $100 \mu \mathrm{l}$ and $200 \mu \mathrm{l}$ of medium without drug, served as growth and medium control respectively. The plates were incubated at $37^{\circ} \mathrm{C}$ for $18 \mathrm{~h}$. The plates were visually read and the minimum concentration of the compound showing no turbidity was recorded as MIC.

\section{Conclusion:}

The main research objective was to unseal the ethnopharmacological value of Angelica archangelica through this new strategy i.e. flash chromatography fractionation. The positive biological activities manifested by the fractionated extracts from crude extracts render concrete substantiation for the use of such species for traditional medicinal purposes. An inquest into these biological activities is well grounded by the fact that pharmaceutical and cosmetic industries are investigating alternative, more natural antimicrobials. Fractions like F3b (A001), F4b (A001), F6a (A001), F3a (A002) and F3a (A003) were the most active fractions out of all the extracts tested. One more interesting result was that all the fractions of ethyl acetate were more active. So these 
fractions can be further investigated phytochemically for better result. Chemical constituents can be isolated from these fractions which may unseal the great medicinal and pharmacological properties of this plant.

\section{References}

[1]. M. M. Cowan, Plant Products as Antimicrobial Agents: Clinical Microbiology reviews, American Society of Microbiology, 1999, 564-582.

[2]. C. A. Newall, L. A .Andersen, J .D. Phillipson. Herbal Medicines A guide of Health Care Professionals . (The pharmaceutical press London 1996).

[3]. Anonymous, The weath of India: a dictionary of Indian raw material and natural products. 1985; 4, p-257

[4]. R.N.Chopa,S.I.Nayal,I.C.Chopra, Glossary of Indian medicinal plants. (New Delhi CSIR, 1956.)

[5] J.Duke, Handbook of medicinal herbs.(Boca Raton, USA: CRC Press Inc.1987)

[6]. O.Polunin, A.Stainton, Flowers of Himalaya. (Oxford University Press, Delhi 1984).

[7] V.S. Agarwal, Economic plants of India.(Kailash Prakashan Culcutta 1986)

[8]. M.R.Uniyal, N.S.Chauhan, Medicinal Plants of Vhat Valley in Kangra forests : J.Res. Indian Med: 1971, $287-299$.

[9]. K.R. Kirtikar, B.D.Basu, Indian Medicinal Plants,Allahabad, 1988, 1214-1215.

[10] R.N. Sharma,S. G.Deshpande, M. Joseph,Evaluation of Natural Essential Oil of Angelica gluca of different geographical regions, Indian Perfumer, 34, 1990, 196-198.

[11] Warren Steck, B. K. Baily, leaf coumarins of Angelica archangelica, Canadian journal of chemistry 47,1969, 2425-2430.

[14] Yong Soo Kwon, Akio Kobayashi, S. I. Kajiyama, Hiroshi Kanzaki, Chang min Kim, Antimicrobial constituents of Angelica dahurica, Phytochemistry 44(5), 1997, 887-889.

[12] Jaroslaw Widelski et al, Coumarins from Angelica lucida and antibacterial activities, Molecules, 14, $2009,2729-2734$.

[13] E. David Wedge,A.K. Jerome, Bioactivity guided fractionation and GC-MS fingerprinting of Angelica sinensis and Angelica archangelica root components for anti fungal and mosquito deterrent activity, J. Agric. Food Chemistry, 57, 2009, 464-470.

[14] S. Sigurdsson, H.M. Ogmundsdottir, \& S. Gudbjarnason, Antiproliferative effects on Angelica archangelica fruits. Z Naturforsch, 59(7-8), 2004:523-527.

[15] J.B.Harborne, T.J. Mabry,H. Mabry,(The Flavonoids.Chapman\& Hall, London, 1975) 